\title{
ANÁLISIS PRELIMINAR DE LA ICTIOFAUNA DE ALTO GUAYACÁN (COSTA RICA) CON BASE EN LOS OTOLITOS. FORMACIÓN USCARI (MIOCENO SUPERIOR-PLIOCENO INFERIOR)
}

\author{
César A. Laurito M. \\ Sección de Paleontología y Estratigrafía, Dirección de Geología y Minas; Ministerio \\ del Ambiente y Energía. Apartado postal 10104-1000, San José, Costa Rica
}

(Recibido 6/8/1996; Aceptado 13/3/1997)

\begin{abstract}
The beds of the well-known fossil mollusc locality of Alto Guayacan contain a rich selachian fauna and an important otolith assemblage. This assemblage illustrates conditions of the outer shelf zone, with normal salinity and direct conection with the open ocean, near mesopelagic zone. The fossil record recovered, comprises: 4 orders, 7 families, 10 genera and at least 18 species. Some genera were reported previously for others geological formations in the Caribbean Region, like Gatun Fm. in the Panama Canal Zone or the Gurabo and Mao Formations in the Pliocene of the Dominican Republic.
\end{abstract}

\begin{abstract}
RESUMEN: La localidad de Alto Guayacán constituye un importante yacimiento de otolitos dentro de la Formación Uscari y en la región del Caribe de América Central Meridonal. La ictiofauna representada por los otolitos, consta de cuatro ordenes, siete familias y diez géneros, para un aproximado de dieciocho especies que aportan datos importantes sobre la batimetría, el habitat y aspectos tróficos. Además, es posible hacer inferencias paleobiogeográficas con otras localidades del Caribe, en especial la Fm. Gatún, en la zona del canal de Panamá y las Fms. Gurabo y Mao en el Plioceno de República Dominicana.
\end{abstract}

\section{INTRODUCCIÓN}

Los amplios depósitos sedimentarios de la Formación Uscari son famosos por sus abundantes faunas de foraminíferos (Pizarro, 1987). Sin embargo, en estas sedimentitas son localmente comunes los moluscos (Olsson, 1922; Aguilar, 1993), dientes tanto de peces cartilaginosos como óseos y otros restos, entre ellos destacan las sagitas del sáculo ótico de los peces óseos, denominados otolitos.

Los otolitos son complejos cuerpos policristalinos, que actúan como órganos del equilibrio o estático-acústico dentro del oído interno. Están constituidos principalmente por carbonato de calcio, cristalizado en forma de aragonito. Los otolitos crecen por aposición de materiales sobre su superficie, esta aposición es cíclica y está en función de los ritmos internos del metabolismo del calcio y de la síntesis aminoácida. Resultando la formación de incremento de crecimiento diario del otolito (Morales-Nin, 1991).

El laberinto de los peces cuenta con tres tipos de otolitos: el asteriscus radicado en la lagena del laberinto, el lapillus en el utrículo y la sagitta en el sáculo, éste último crece mucho más que los dos restante (con la excepción de los ostariofisios no gonorhynchiformes) y es básicamente el que se utiliza en los estudios micropaleontológicos por su alto potencial de 
fosilización, su valor taxonómico e incluso filogenético.

El gran valor paleoecológico, deriva del interés económico que reviste la industria pesquera actual, ya que el conocimiento del ciclo vital y la ecología de los peces tiene una larga trayectoria de estudio, cuyas concluciones es posible extrapolar al pasado (Nolf, 1995).

El objetivo del presente trabajo es dar a conocer de manera preliminar, la primera asociación paleoictiológica del país, basada en otolitos y dar algunas reseñas paleoambientales del sitio Alto Guayacán.

\section{METODOLOGÍA}

Los 865 otolitos hasta ahora analizados, fueron obtenidos en su mayoría directamente del afloramiento, otros fueron entresacados de concentrados fosilíferos, previamente preparados. Los concentrados se consiguieron a partir del procesamiento de cerca de $300 \mathrm{~kg}$ de sedimento, a la que se le eliminó la arcilla y la fracción de limo. Luego con la ayuda de un miscroscopio estereoscópcio o a simple vista se separaron los especímenes y se limpiaron en una cubeta ultrasónica. Posteriormente los otolitos se agruparon por topologías y se procedió a su determinación preliminar.

\section{UBICACIÓN DEL YACIMIENTO}

El afloramiento se ubica en el caserío de Alto Guayacán, aproximadamente $10 \mathrm{~km}$ al Oeste de Siquirres (Fig. 1), provincia de Limón, en las coordenadas Lambert Costa Rica Norte 225.2 $\mathrm{N}$ y 585.65 E Hoja topográfica Bonilla, IGN-CR escala 1:50 000 .

\section{EDAD Y POSICIÓN ESTRATIGRÁFICA}

La Formación Uscari ha sido ampliamente estudiada por diversos autores (en años recientes Pizarro, 1987; Fenández, 1987; Campos, 1987, Cervantes, 1989, Botazzi et al., 1994, Sprechmann et al. 1994). Esta formación comprende importantes afloramientos en la Cuenca Limón Sur. El espesor de sus sedimentos alcanza el orden de los 1000 metros y su edad es diacrónica variando desde el Mioceno inferior hasta el Plioceno inferior de acuerdo al presente trabajo.

Las litologías predominantes son arcillolitas y limolitas, de color gris azul a gris verdoso, con estratificación homogénea de escala decimétrica, métrica hasta masiva, incluyendo frecuentes intercalaciones de areniscas de grano fino, calcilutitas y lentes de material conglomerádico.

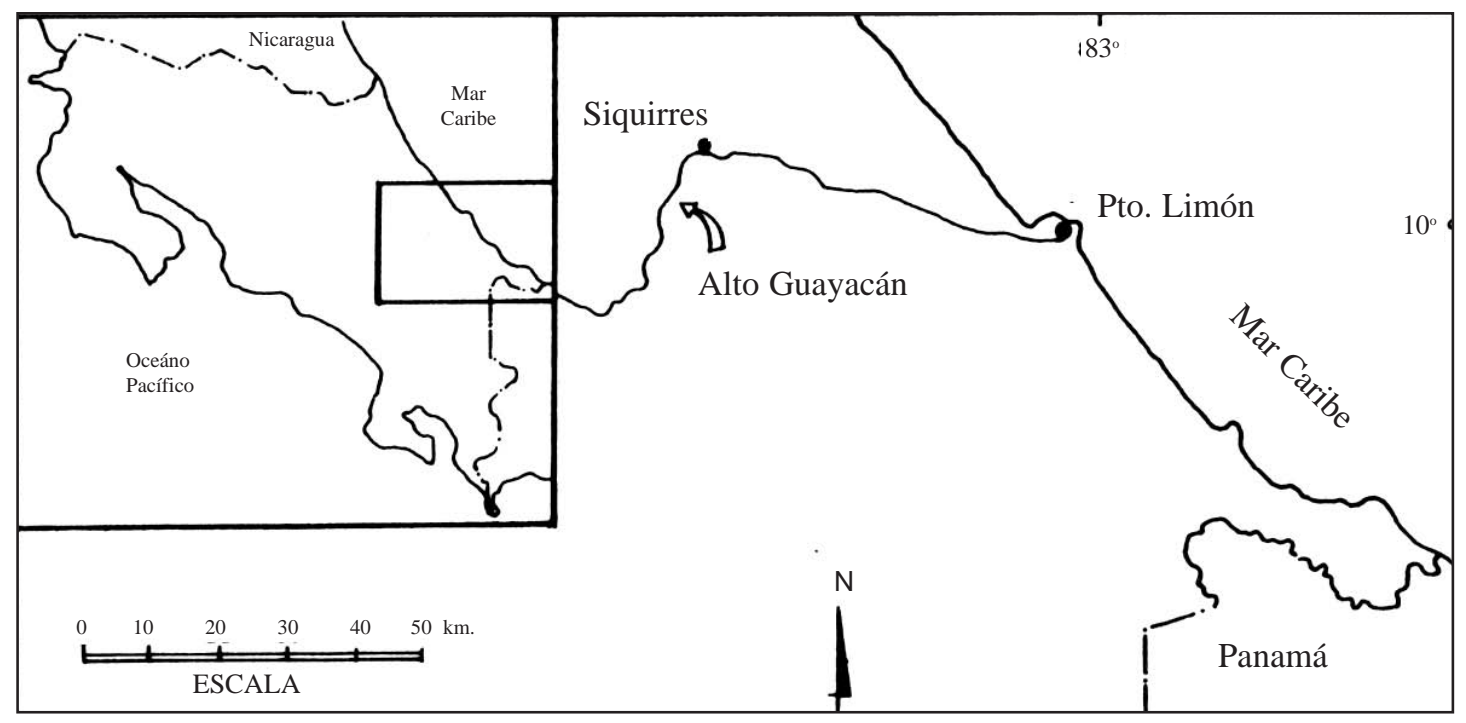

Fig. 1. Ubicación de la localidad de Alto Guayacán. 
En el afloramiento de Alto Guayacán, la estratificación varía de decimétrica a métrica, predominan los estratos arcillosos, pero existen intercalaciones de limolitas que varían a arcillas, con fuerte bioturbación; son comunes los lentes de amplitud decimétrica y longitud métrica de arenas medias y gruesas. El tope de esta secuencia está dominado por calcarenitas medias, fuertemente cementadas que forman bancos métricos, indentándose con estratos arcillolíticos. Finalmente el perfil culmina con flujos de lava, a manera de relictos.

De acuerdo con la columna estratigráfica (Fig. 2), las muestras para micropaleontología y de otolitos se tomaron de los niveles A al E, que representan la parte baja del corte. La diversidad faunística se mantuvo constante entre los niveles, al igual que la edad.

La edad del perfil de Alto Guayacán, se obtuvo a partir del análisis de la fauna de foraminíferos planctónicos, realizada por la Licda. Pizarro del Laboratorio de Micropaleontología de RECOPE, para ello se tomaron cuatro muestras, con los siguientes resultados:

Muestra CLM-447, nivel B; edad: Plioceno Inferior.;

Biozona N 19.

Muestra CLM-448, nivel C;

edad: Mioceno Superior - Plioceno Inferior.;

Biozona N 17-19.

Muestra CLM-449, nivel D;

edad: Mioceno Superior - Plioceno Inferior;

Biozona N 18-19.

Muestra CLM-450, nivel E;

edad: Mioceno Superior - Plioceno Inferior.;

Biozona N 17-19.

\section{PALEONTOLOGÍA Y SISTEMÁTICA}

Basicamente se utiliza la sistemática propuesta por Nolf (1985 [non vide]) y que cita en obras posteriores, p.e. Nolf $(1991,1993)$ y otros. La sistemática de grupos suprafamiliares, está basada en el apendice de la clasificación de los vertebrados de Carroll (1987).

Clase OSTEICHTHYES Subclase ACTINOPTERYGII
Infraclase NEOPTERYGII

Division TELEOSTEI

Subdivisión EUTELEOSTEI

Superorden SCOPELOMORPHA

Orden MYCTOPHIFORMES

Familia MYCTOPHIDAE Gill, 1893.

Género Diaphus Eigenmann \& Eigenmann, 1890.

Diaphus es el género más especioso de los myctófidos, sus otólitos se encuentran con frecuencia y son excelentes indicadores batimétricos, especialmente cuando está asociado a faunas batiales.

Sin embargo, son otolitos muy pequeños, delgados y frágiles, que no soportan gran transporte y pueden facílmente perder sus estructuras diagnósticas durante procesos digestivos.

Los otolitos de Diaphus, fueron recolectados en su mayoría directamente del afloramiento en el campo, ya que en los preparados de concentrados fosilíferos, por lo general se disolvían o se fracturaban.

Se hallaron varias morfologías, que pueden corresponder a variaciones ontogénicas o de especie, ello se dilucidará cuando se hagan más comparaciones.

\section{Diaphus "tipo 1"}

Material: 20 ejemplares (lámina 3, Figs. 3a-c).

Es un otolito con excisura recta, el anterostrum se encuentra ligeramente más atrás con respecto al rostrum; posee reducidos domos ventrales a manera de espinas. Perfil cóncavo-convexo.

\section{Diaphus "tipo 2"}

Material: 1 ejemplar (lámina 4, Figs. 1a-b).

Es un otolito con la excisura curva, el rostrum recto y tiene una posición más anterior con respecto al anterostrum; el borde ventral tiene abundantes domos ventrales.

\section{Myctophidae: Género indeterminado}

Material: 4 ejemplares (lámina 4, Figs. 2a-b).

Es un otolito de pequeño tamaño, lo que podría tratarse de un estadio ontogénico temprano o de una especie diferente de Diaphus, por ejemplo pertenecer al género Myctophum. 


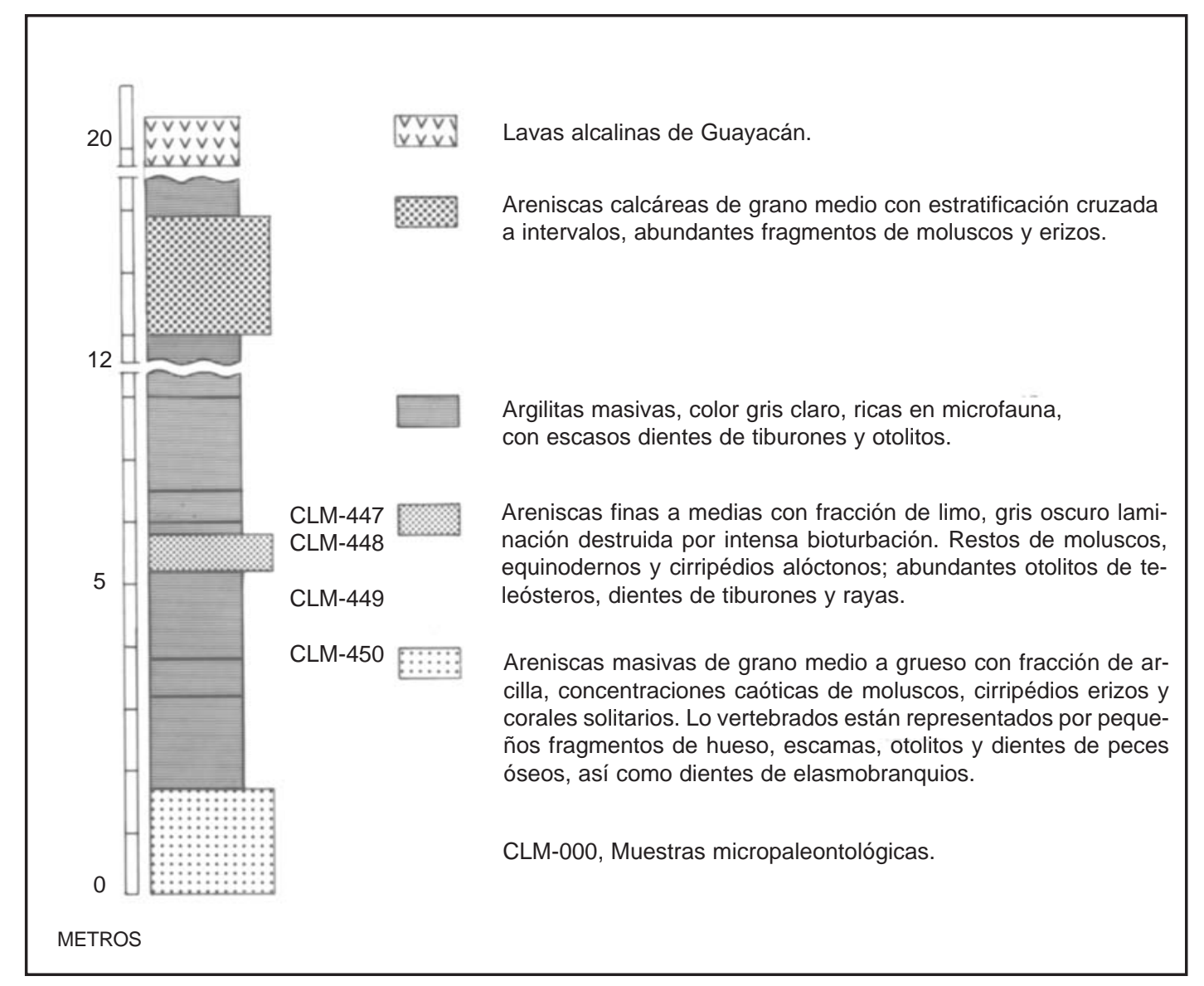

Fig. 2. Columna estratigráfica de la localidad de Alto de Guayacán.

Se caracteriza por poseer un colliculum anterior que se ensancha hacia la excisura, rostrum de posición mucho más externa que el anterostrum, un dorso redondeado, cinco domos ventrales y un perfil plano convexo.

Distribución: Otolitos de myctófidos se han encontrado fósiles en el Mioceno Medio de Panamá, en la Formación Gatún (Gillette, 1984); Mioceno y Plioceno de República Dominicana (Nolf \& Stringer, 1992); Mioceno de México y Jamaica (sensu Fitch, 1969).

Fuera del Caribe, se han encontrado en Lee Creek Mine, North Carolina (Formación Yorktown, Plioceno) sensu Fitch \& Lavenberg (1983), en el Pleistoceno de Playa del Rey y Pleistoceno Inferior de San Pedro, Palos Verdes y Timm Points en California (Fitch, 1966, 1967 y 1969, respectivamente), Pleistoceno TardíoHoloceno Temprano del Delta de Mississipi en Lousiana (Stringer, 1992) y en muchos lugares de Europa (Anfossi \& Mosna, 1973; Nolf \& Steurbaut, 1987; Brzobohaty, 1994; Brzohobaty \& Nolf, 1995).

\section{Superorden PARACANTHOPTERYGII Orden OPHIDIIFORMES Familia OPHIDIIDAE (incl. Brotulidae)}

Los más antiguos otolitos de la familia Ophidiidae datan del Cretácico Superior (Nolf, 1980). 
Género Neobythites Goode \& Bean, 1885

Los otolitos pertenecientes a este género son sumamente abundantes en el afloramiento de Alto Guayacán, diferenciándose dos especies:

\section{Neobythites sp.1}

Material: 144 ejemplares (lámina 2, Figs. 3a-b).

Estos especímenes son morfológicamente muy similares a los de Neobythites marginatus Goode \& Bean, 1886.

$\mathrm{Su}$ aspecto general es de contorno oval elongado y ligeramente poligonal en relación a los bordes dorsal, anterior y posterior; mientras que el borde ventral es convexo.

La cresta superior tiene una suave curvatura, que le da un aspecto casi plano, en tanto que la cresta inferior posee una hendidura poco marcada que divide la cauda del ostium. El otolito en vista de perfil es plano convexo, la excisura del ostium es angosta y se abre al nivel de la cresta superior. El colicullum caudal tiene aproximadamente la mitad de la longitud del culliculum del ostium, el ancho de ambos es muy similar.

\section{Neobythithes sp.2:}

Material: 142 ejemplares (lámina 2, Figs. 4a-b).

Estos especímenes son morfológicamente más similares a la especie Neobythites gillii Goode \& Bean, 1886. Su aspecto general es oval, con bordes redondeados y la cara externa es fuertemente convexa dándole un aspecto inflado; en perfil es plano convexo.

\section{Distribución paleogeográfica:}

Otolitos de este género se han registrado en el Mioceno Temprano de la Formación Brasso en Trinidad (Nolf, 1980 in Nolf \& Stringer, 1992), en la Formación Mao del Plioceno de República Dominicana y la Formación Yorktown del Plioceno de North Carolina (Fitch \& Lavenberg, 1983; Figs. 2D y 2E).

\section{Género Brotula Cuvier, 1829}

\section{Brotula sp.}

Material: 9 ejemplares (lámina 2, Fig. 1a-b).

Los ejemplares recolectados de Brotula son morfológicamente similares a los de Brotula barbata (Bloch \& Schneider). Estos otolitos se caracterizan por ser fusiformes, delgados y ligeramente sinuosos. Su morfología típica permite una fácil identificación.

\section{Distribución Paleogeográfica:}

La especie Brotula barbata que actualmente vive en el Atlántico, se ha registrado en el Mioceno de Trinidad (Nolf, 1976 in Fitch \& Lavenberg, 1983), en el Plioceno de Yorktown, North Carolina (Fitch \& Lavenberg, 1983; fig. 2a) y especímenes relativos a la especie Brotula clarkae Hubbs, 1944 se han descrito para las Formaciones Gurabo del Plioceno Inferior y Mao del Plioceno de República Dominicana (Nolf \& Stringer, 1992).

\section{Género Lepophidium Gill, 1895 Lepophidium sp.}

Material: 292 ejemplares (lámina 2, 2a-b).

Los ejemplares recolectados de Lepophidium son morfológicamente similares a la especie Lepophidium cervinum (Goode \& Bean) y constituyen el grupo de otolitos más abundante hallado en la Fm. Uscari. Se caracterizan por poseer un conspicuo proceso espiniforme posterior, situado ligeramente por debajo del plano ecuatorial del otolito, en algunos ejemplares pareciera que el proceso espinoso se sitúa marcadamente más abajo.

Su cara ventral es muy inflada, a veces presenta irregularidades que llegan a formar verdaderos apéndices diferenciables, pero por lo general la superficie ventral es ligeramente irregular y suave. También posee un apéndice que se proyecta desde la superficie ventral hacia una posición anterior, que en vista dorsal sobresale por el margen dorsal, arriba del ostium. La cauda es redondeada y el ostium es alargado, la incisión del sulcus es muy somera, el contorno del plano dorsal es oval y ligeramente convexo. Los collicula forman un continuo, no siendo posible diferenciarlos.

\section{Distribución paleogeográfica:}

La especie Lepophidium latesulcatum Nolf \& Stringer, 1992 se ha desrito en las formaciones Mao y Gurabo del Plioceno y las formaciones Baitoa del Mioceno Temprano y Medio y Cercado del Mioceno Superior, todas de la República Dominicana.

La especie Lepophidium cf. cervinum, resulta ser la más abundante de Lee Creek Mine del Plioceno de North Carolina (Formación Yorktown, de acuerdo a Fitch \& Lavenberg, 1983; fig. 2B) y Lepophidium cervinum ha sido registrado en el Pleistoceno Tardío-Holoceno 
Temprano del Delta del Mississippi en Lousiana (Stringer, 1992).

La especie Lepophidium negropinna ha sido registrada en el Pleistoceno de Playa del Rey y San Pedro en California (Fitch, 1964 y 1970).

\section{Género "Dinematichthyinorum"} b y $2 a-b)$.

Material: 25 ejemplares (lámina 3, Figs 1a-

Es un género nominal, relacionado al los géneros Brotulina y Dermatopsis de acuerdo con Nolf, 1980 y Nolf \& Stringer, 1992. Sin embargo, morfológicamente los otolitos de éste género son muy similares a los descritos por Fitch (1967, fig. 6; 1968 fig.3c) como pertenecientes Brosmophycis marginata un Brotulidae.

Son otolitos ovales y elongados con extremos agudos y de pequeñas dimenciones, cuya morfología es comparable a un grano de arroz.

Dentro del conjunto recolectado en Alto Guayacán, se observan topologías variables, que pueden explicarse como tipos ontogénicos o especies diferentes; sin embargo, para esclarecer esta cuestión es necesario hacer más comparaciones.

Distribución paleogeográfica: Dos especies asociadas a este género fueron recolectas en las formaciones Cercado, Gurabo y Mao (Mioceno Tardío, Plioceno Temprano y Plioceno, respectivamente) de República Dominicana.

Fuera del Caribe, éste género se ha registrado en Timms Point Silt (Pleistoceno Temprano) en San Pedro, California y en diversos puntos en depósitos del Plioceno y Pleistoceno del Sur de California (Fitch, 1967 y 1968). También, en el Paleoceno de Alabama (Nolf \& Dockery, 1993).

\section{Subdivision ELOPOMORPHA Orden ANGUILLIFORNES Familia CONGRIDAE}

Género Ariosoma Swainson, 1838.

\section{Ariosoma sp.1.}

Material: 3 ejemplares (lámina 1, Figs. 1a-b).

Los otolitos de esta especie son morfológicamente cercanos a Ariosoma balearica (Delaroche, 1809). Son otolitos poco frecuentes, su forma semeja un rombo, con el borde dorsal formando una prolongación o domo dorsal (sensu Frizzell \& Lamber, 1962), el sulcus relativamente ancho, con una excisura que alcanza el borde dorsal por encima del nivel de la cresta superior y es sinuosa. No se diferencian los collicula.

\section{Ariosoma sp. 2.}

Material: 73 Ejemplares (lámina 1, Figs. 2a-b).

Los otolitos de esta especie se diferencian de la anterior por ser más achatados u ovales, el domo dorsal no ocupa una posición mediana, sino, se encuentra desplazado hacia la porción ostial. Su contorno es uniforme. El tamaño es la mitad del alcanzado por la especie 1 .

\section{Distribución:}

La especie Ariosoma balearica (Delarroche, 1809) aparece en las formaciones Baitoa (Mioceno Temprano y Medio); Cercado (Mioceno Tardío); Gurabo (Mioceno Tardío-Plioceno Inferior) y Mao del Plioceno; todas de la República Dominicana. La especie Ariosoma aff. $\boldsymbol{A}$. selenops Reid, 1934, en las formaciones Cercado y Mao (Nolf \& Stringer, 1992).

Fuera del Caribe, se ha descrito recientemente en el Eoceno de Pakistan (Nolf, 1991) y la especie Ariosoma moravica se ha descrito en el Badeniano Inferior (Mioceno Medio) de los Carpatos orientales (Brzobohaty, 1983).

\section{Género Rhynchoconger}

Material: 44 ejemplares (lámina 1, Figs. 3a-b).

Los ejemplares pertenecientes a éste género son de forma oval ligeramente elongado, su contorno ventral es uniformemente curvo, mientras que el contorno dorsal es más plano, con sendas convexidades irregulares hacia las porciones ostial y posterior; presenta una región endida arriba de la cresta superior o área (sensu Frizzell \& Lamber, 1962).

\section{Distribución:}

La especie Rhynchoconger flavus (descrita como Hildebrandia flava cf. Nolf \& Dockery, 1993) se ha encontrado en las formaciones Gurabo y Mao (Nolf \& Stringer, 1992).

Fuera del Caribe el género Rhynchoconger se ha registrado en el Plioceno de Yorktown, North Carolina (Fitch \& Lavenberg, 1983; fig. 1C, como Congridae especie B), en el Paleoceno de Alabama en la Formación Porters Creek (Nolf \& Dockery, 1993), Pleistoceno Tardío-Holoceno Temprano del Delta del Mississippi en Lousiana (Stringer, 1992; quien lo 
registro como Hildebrandia); en el Oligoceno Tardío de Hungría en la Formación Eger (Nolf \& Brzobohaty, 1994) y en el Mioceno Medio de Austria en la localidad de Gainfarn (Brzobohaty, 1994).

\section{Género Rechias}

Material: 95 ejemplares (lámina 1, Figs. 4, 5a-b). Otolitos de este género son de pequeño tamaño, ovales con el contorno inferior redondeado y uniforme, contorno dorsal con la porción anterior convexa a veces recta y porciones superior y posterior casi rectas y contactos angulosos. Los cullicula no están diferenciados; presenta área.

\section{Distribución:}

La especie Rechias tysanochila (Reid, 1934) se ha hallado en las formaciones Cercado y Mao (Mioceno Tardío y Paleoceno respectivamente) en República Dominicana (Nolf \& Stringer, 1992).

Fuera del Caribe el género Rechias se ha registrado en la Formación Kiscell del Oligoceno Tardío de Hungría (Nolf \& Brzobohaty, 1994) $\mathrm{y}$ en el Plioceno Inferior de Lugagnano, Piacenza en Italia (Anfossi \& Mosna, 1973).

Nota: Ophididae indeterminados han sido descritos para el Mioceno Medio de la Formación Gatún en Panamá (Gillette, 1984).

\section{Superorden ACANTHOPTERYGII Serie PERCOMORPHA \\ Orden PERCIFORMES Familia SCIAENIDAE}

Material: 2 otolitos (lámina 5, Figs. 2a-b y 3a-b). Las corvinas están representadas por dos otolitos de morfología característica, pero que por el momento no han podido ser identificados con exactitud por falta de material comparativo.

\section{Distribución:}

Los otolitos de las corvinas son frecuentes, en casi todos los depositos sedimentarios neríticos y se sabe que muchas especies alcanzan hasta profundidades cercanas a los 300 metros.

\section{Familia POMADASYDAE}

\section{Género Isacia}

Material: 7 ejemplares (lámina 5, Figs. 2a-b)

Los otolitos asociados a éste género son ovales elongados de aproximadamente $3 \mathrm{~mm}$ de largo, de aspecto ligeramente trapezoidal, pero de contorno redondeado, presenta "área", el rostrum es redondeado y de posición más anterior que el anterostrum, colliculum anterior oval, la cauda es elongada y suavemente curva.

\section{Distribución:}

El género Isacia se ha registrado en la Formación Wittering en Inglaterra, y en el Miembro Egem Sand en Bélgica, ambas del Ypresiano (Sterbaut \& Nolf, 1990; pág. 333). En la actualidad la especie Isacia conceptionis $(\mathrm{Cu}-$ vier) se distribuye en el océano Pacífico entre los $6^{\circ}$ y $37^{\circ}$ de latitud sur, frente a las costas de Perú y Chile (Chirichigno, Fischer \& Nauen, 1982; pág. 190).

\section{Familia ACROPOMATIDAE}

\section{Género Acropoma}

Material: 3 ejemplares (lámina 5, Fig. 1a-c)

Son otolitos relativamente pequeños, de contorno oval con domos dorsales poco prominentes. El culliculum anterior es ovalado y más pequeño que el culliculum posterior que es elongado al igual que la cauda, se observa surco ventral y el borde ventral es hiperbólico. La excisura es angulosa y el rostro tiene una posición muy distal en relación con la excisura. En vista de perfil el otolito es cóncavo convexo con una protuberancia central en el lado interno.

Los otolitos de Alto Guayacán asociados al género Acropoma, se diferencian difícilmente de la especie Acropoma japonica, que habita en el océano Pacífico.

\section{Distribución:}

El género nominal "Acropomatidarum", que por su morfología se considera cogenérico de Acropoma se ha registrado en la Formación Kiscell Clay del Oligoceno Temprano de Hungría (Nolf \& Brzobohaty, 1994; pág. 234)

\section{Familia Indeterminada}

Material: 1 otolito (lámina 4, Fig.3).

Otolito de pequeño tamaño, que morfológicamente y de manera preliminar se asocia a un Gadiforme.

\section{Paleoecología}

El análisis preliminar con base en los otolitos de Alto Guayacán, nos muestra una asociación 
faunal ictiológica compuesta hasta ahora de seis familias, a saber:

Familia Myctophidae: Son pequeños peces mesopelágicos y batipelágicos (Chirichigno, Fischer \& Nauen, 1982) que viven principalmente en la zona comprendida entre los 200 y 1000 metros de las aguas océanicas. La mayoría de las especies de esta familia efectúan migraciones verticales diarias, la noche es el momento en que incluso pueden alcanzar la superficie, para luego descender de la zona eufótica a más de 200 metros.

La abundancia de estos peces en la zona mesopelágica es proporcional a la abundacia de sus otolitos en depósitos oceánicos asociados a dichos ambientes. Esta condición se mantiene para áreas neríticas abiertas al dominio oceánico. De ahí la gran importancia estratigráfica de los Myctophidae (Brzobohaty \& Nolf, 1995).

Familia Ophididae: Son peces tropicales y de aguas templadas, que generalmente habitan aguas profundas. Algunas especies viven en aguas costeras en pozos de marea y esteros. Otros se entierran en la arena (Bussing \& Lopez, 1993).

El género Brotula tiene un habitat bentónico de plataforma (fondos blandos) y bentónico de talud (Chirichigno, Fischer \& Nauen, 1982).

El género Neobythites tiene un habitat bentónico de plataforma al igual que el género Lepophidium (fondos blandos) (Cervigón \& Fischer, 1979)

Familia Congridae: Viven en aguas costeras poco profundas, tropicales y semi-templadas, aunque se creen bajan a aguas más profundas para reproducirse (sensu Bussing \& Lopez, 1993); bentónicos de plataforma y del talud (Chirichigno, Fischer \& Nauen, 1982).

Los géneros Ariosoma, Rhynchoconger y

Rechias son bentónicos de plataforma.

Familia Sciaenidae: Son peces principalmente de aguas tropicales o semitempladas y habitan aguas costeras, esteros y aguas dulces (Bussing \& López, 1993), se cree alcanza los 300 metros de profundidad para reproducirse.

Familia Pomadasydae: Se caracteriza por especies bentónicas de plataforma, algunas frecuentan estuarios y por lo general sobre fondos duros, el género Isacia es bentónico pelágico y pelágico costero.

Familia Acropomatidae: El género Acropoma habita la región mesopelágica (bajo los 200 metros hasta más de 500 metros).

Fundamentándose en los datos recolectados hasta ahora, se concluye la siguiente interpretación paleoambiental:

a. Batimetría: De acuerdo con la figura 3, la distribución batimétrica actual de taxa de teleósteos representados en la asociación de otolitos de Alto Guayacán, permite concluir provisionalmente que la profundidad de coincidencia del 100\% de las especie es cercana a los 200 metros, ello podría variar con el hallazgo de nuevos taxa.

Lo anterior está en concordancia con la relación entre la abundancia de peces neríticos/myctófidos/macrúridos expuesta por Nolf \& Brzobohaty (1992). En este caso y hasta el momento los macrúridos no han aparecido, por lo que se descarta una batimetría mayor.

b. Luz: La presencia de la familia Myctophidae, conocidos como peces linternas, su habitat Meso-batipelágico, su relativa abundancia, más el hecho de que los Ophididae y Congridae, suelen habitar y visitar las plataformas por debajo de la zona eufótica y que el género Acropoma habita bajo los 200 metros de profundidad, es muy probable que el ambiente se caracterizara por la escacez de luz, lo que sugiere una zona de penumbra o zona crepuscular.

c. Salinidad: No cabe duda de que la asociación ictiológica de Alto Guayacán, representa una salinidad cercana a la normal.

d. Sustrato: La predominacia cuantitativa de otolitos de Ophididae y Congridae, cuyos géneros Lepophidium, Brotula, Neobythites y Ariosoma, Rechias, y Rhynchoconger respectivamente, representan más del 75\%, habitan enterrados en fondos suaves (lodosos y arenosos). Lo anterior se comprueba por la predominacia arcillosa de la litología. 
e. Oxigenación: La abundancia de planctón (principalmente foraminíferos), gran cantidad de especies de tiburones y batoideos (incluso algunos de ellos de habitos plantónicos), abundantes restos de peces óseos (fuera de los otolitos), sugiere aguas ricas en oxígeno y en nutrientes.

\section{CONCLUSIONES}

La asociación de peces óseos de Alto Guayacán, es la primera de este tipo estudiada en el país y está representada principalmente por otolitos, minoritariamente por piezas dentales y por otros ictiorodulitos (vertebras y escamas).

Los dientes comunmente hallados, en el caso de los peces óseos corresponden a especies predadoras cuyos otolitos no están representados en el registro fósil o que hasta el momento no se han encontrado. Los restantes dientes, pertenecen a condrictios o peces cartilaginosos que no poseen otolitos.

Los otolitos constituyen el segundo grupo de vertebrados más importante de Alto Guayacán, el primer lugar lo ocupan los dientes de condrictios. Lo anterior puede representar una relación directa entre el porcentaje de predadores y la abundancia de otolitos encontrados.
La interpretación paleoecológica se ha formulado con base en la comparación del habitat de los taxa actuales, lo que ha permitido determinar de manera preliminar que el escenario de Alto Guayacán corresponde a un ambiente mesopelágico superior, próximo al borde de la plataforma y expuesto a la influencia oceánica.

Por último, cabe destacar la importancia que esta asociación paleoictiológica reviste para el entendimiento de la evolución ambiental y paleobiogeográfica del Caribe, en especial en lo que al cierre de América Central Meridonal se refiere ya que de acuerdo a las edades obtenidas con base en forminíferos plantónicos, rango de Biozonas N-17 a N-18 (Mioceno Superior cuspidal-Plioceno Inferior basal), es posible establecer este evento con exactitud espacial y temporal, mismo que a futuro podrá contextualizarse y correlacionarse con nuevos hallazgos dentro de la región del Caribe.

\section{AGRADECIMIENTOS}

Al Dr. Dirk Nolf del Departamento de Paleontología del Real Instituto de Ciencias Naturales de Bélgica y al Dr. Rostilav Brzobohaty del Instituto de Geología y Paleontología de la Universidad de Masaryk (Brno), República Checa,

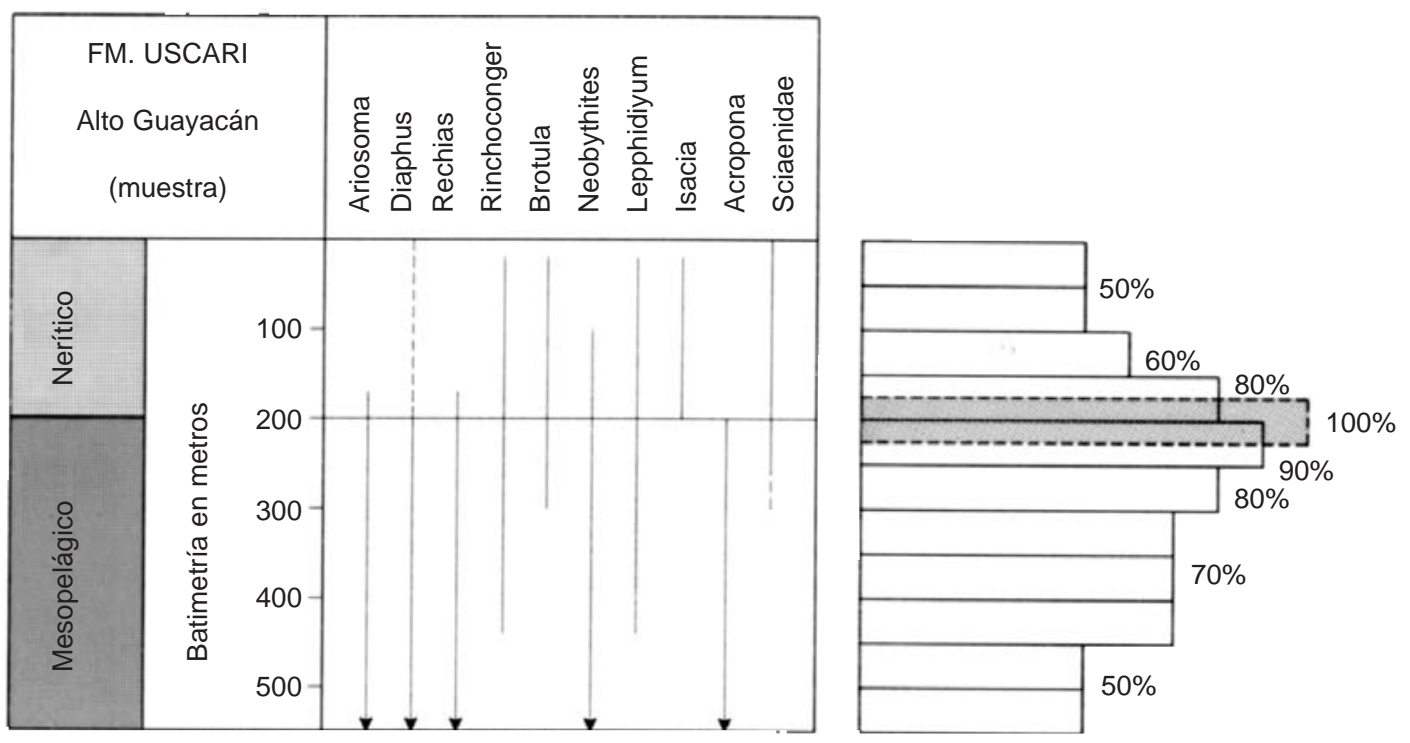

Fig. 3. Gráfico de distribución batimétrica de los géneros actuales representados en la fauna fósil de Alto Guayacán. 


\section{LÁMINA $\mathbf{N}^{\circ} 1$}

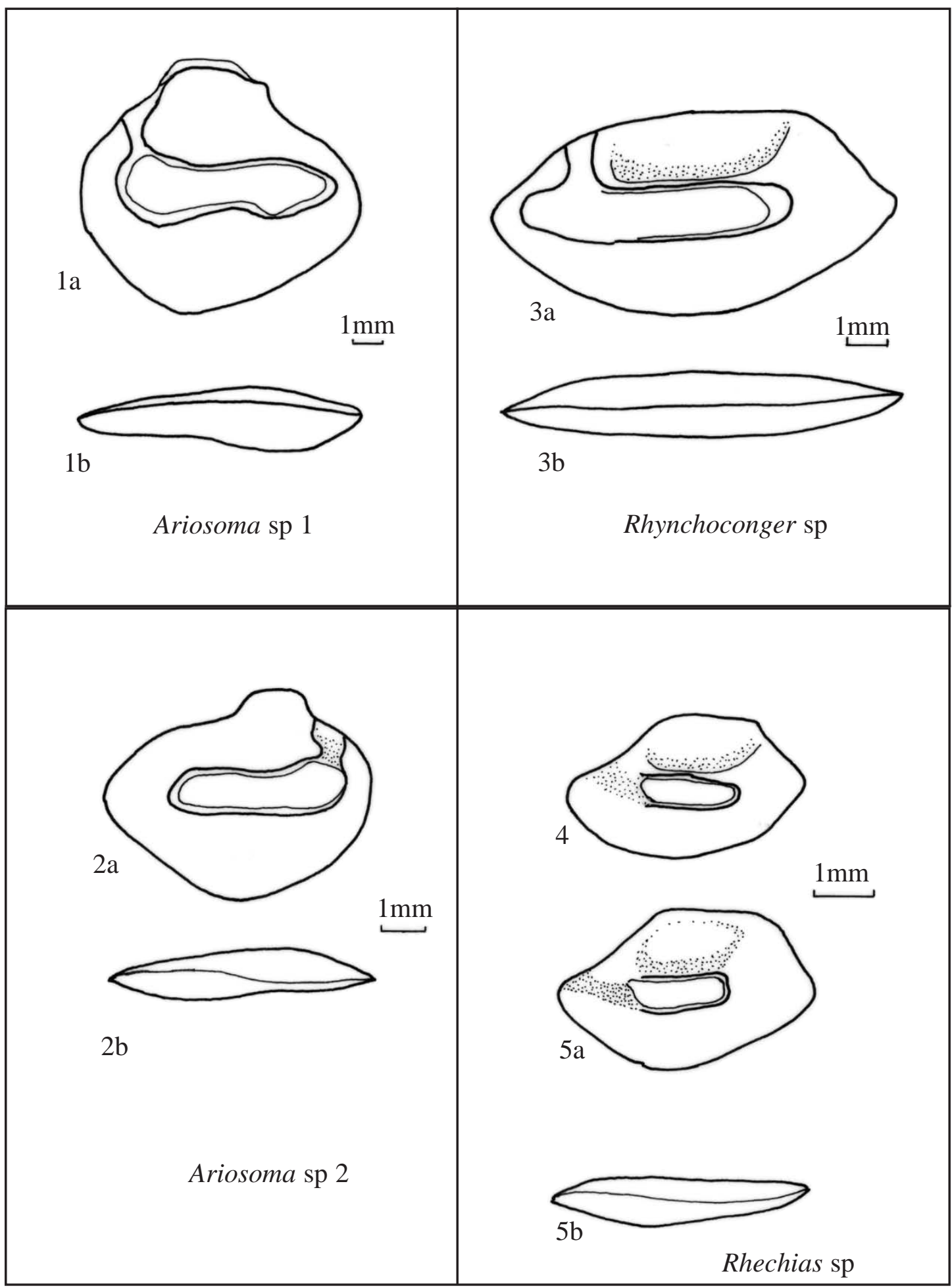




\section{LÁMINA $\mathbf{N}^{0} 2$}

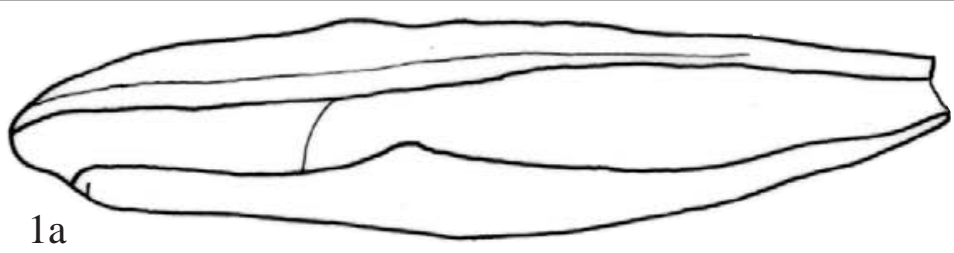

$1 \mathrm{~mm}$

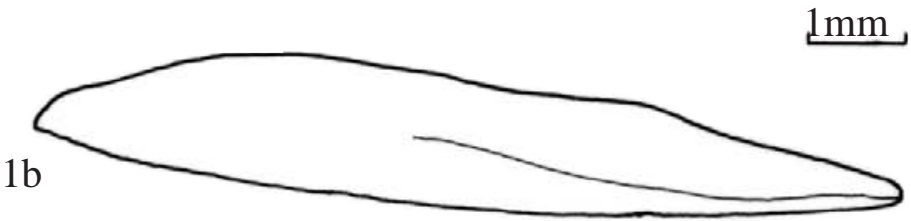

Brotula sp
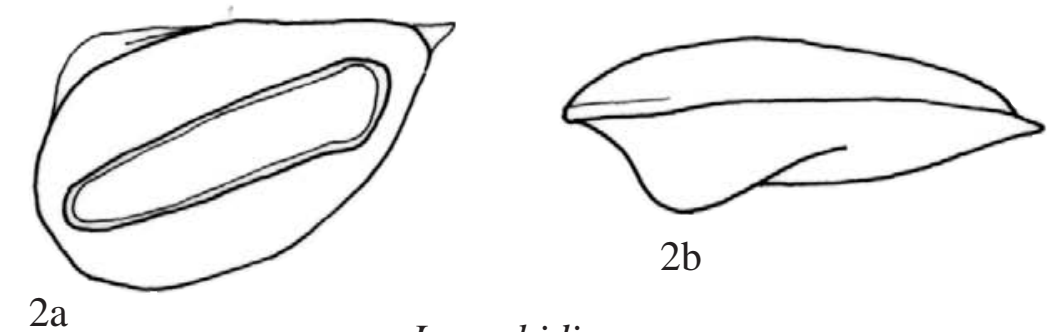

Lepophidium sp
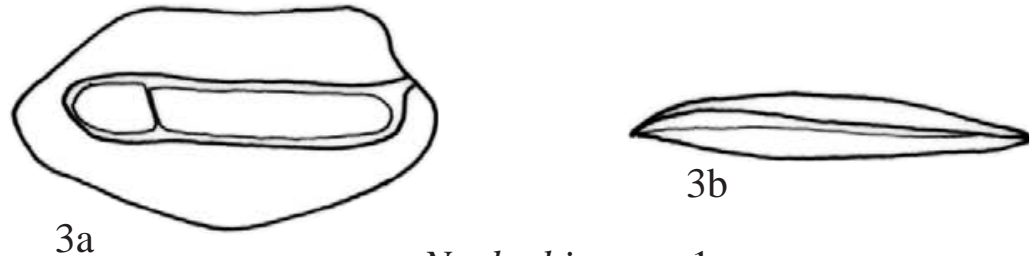

Neobythites sp 1
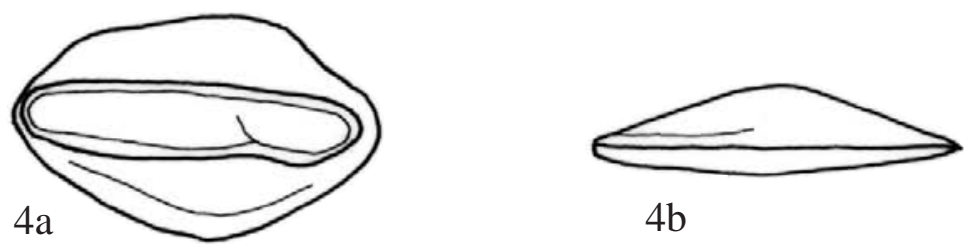

$4 \mathrm{~b}$

Neobythites sp 2 


\section{LÁMINA No 3}

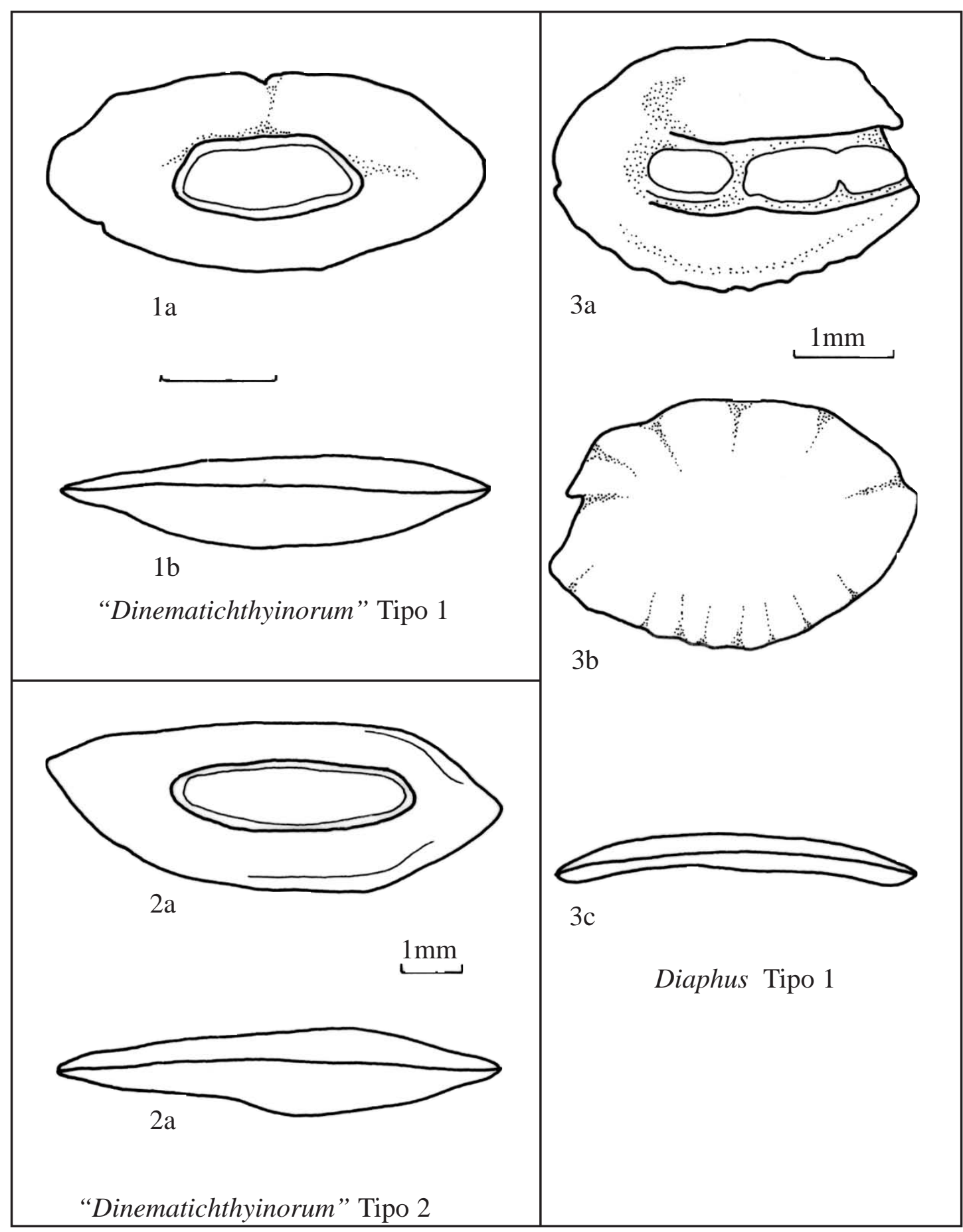




\section{LÁMINA No 4}
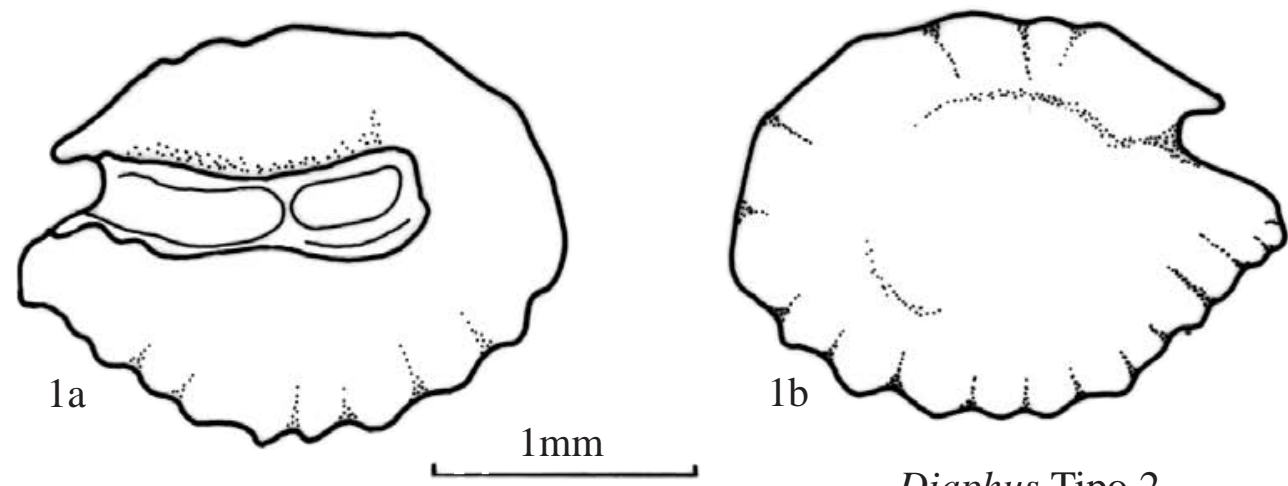

Diaphus Tipo 2
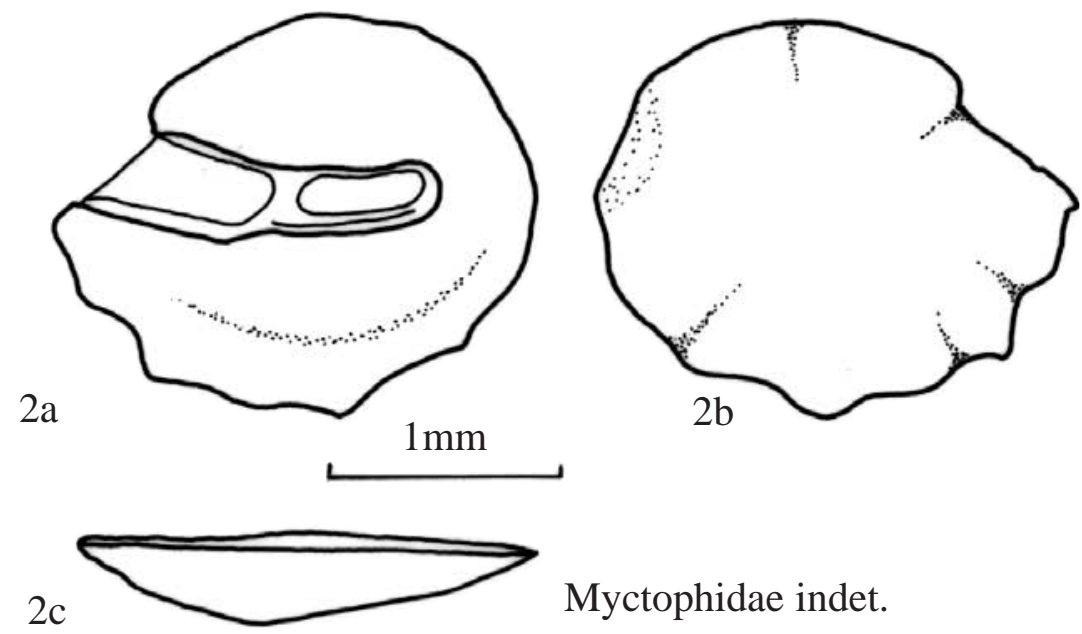

3

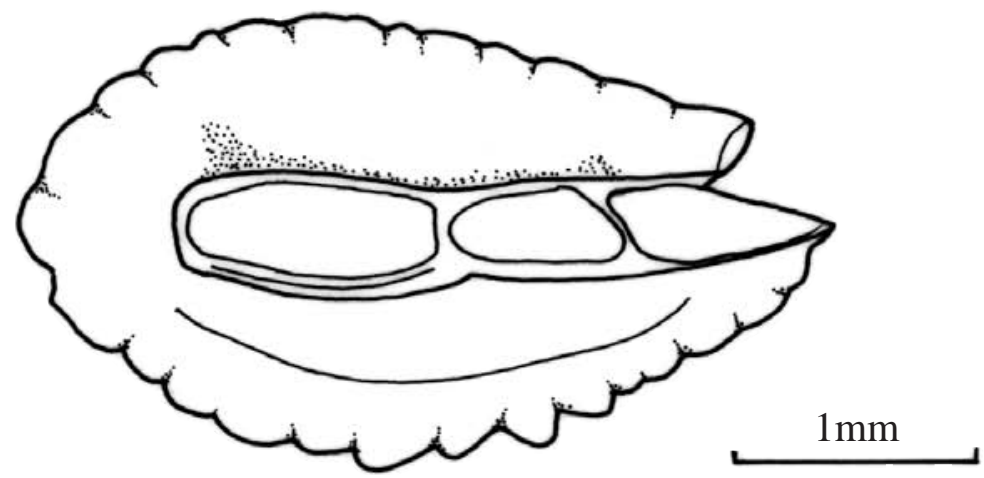

Familia indet. 


\section{LÁMINA No $^{\circ}$}

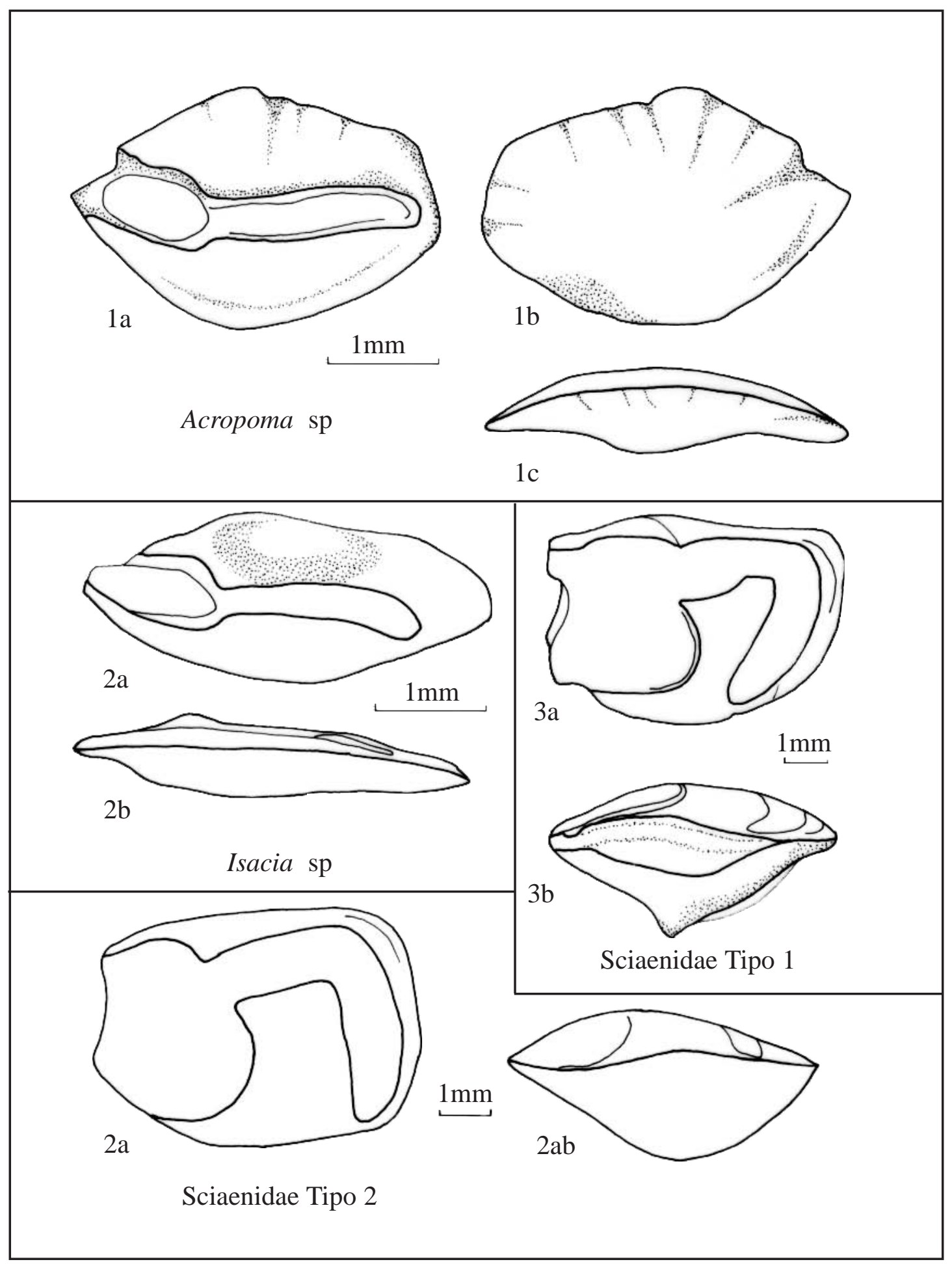


por el interés mostrado en ésta investigación y el amplio apoyo bibliográfico.

A la M. Sc. Marlene Salazar, Directora de la Dirección de Geología y Jefa del Departamento de Planificación y Desarrollo (D.G.M.), quien apoyó la realización de ésta investigación. A la Lic. Diana Pizarro del Laboratorio de Micropaleontología de RECOPE,quien gentilmente realizó los análisis de laboratorio y obtención de las dataciones.

Mi especial reconocimiento al Sr. Carlos Ramírez de la Unidad de Cartografía Digital de la D.G.M. por la elaboración de las figuras y al Téc. Eduardo Pérez Gamboa por la asistencia en las labores de campo y laboratorio.

\section{BIBLIOGRAFÍA}

AGUILAR, T., 1993: Paleoecología de Alto Guayacán Formación, Uscari, Mioceno, Provincia de Limón, Costa Rica. - Rev. geol. América Central, 16: 51-60.

ANFOSSI, G. \& MOSNA, S., 1973: Otoliti del Pliocene Inferiore di Lugagnano (Piacenza). - Atti Ist. Geol. Univ. di Pavia, 23: 90118.

BOTAZZI, G., FERNÁNDEZ, A. \& BARBOZA, G., 1994: Geología e historia tectonosedimentaria de la Cuenca Limón Sur. En: Seyfried \& Hellmann (eds.): Geology of an evolving island arc. - Profil, 7: 351389.

BRZOBOHATY, R., 1983: Fish otoliths from West Carpathian Tertiary and their biostratigraphical significance. - Knihovnicka Zemniho plynu a nafty, 4: 247-266.

BRZOBOHATY, R., 1994: Die Fischotolithen des Badenien von Gaifarn, Niederösterreich (Mittelmiozän, Wiener Becken). Ann. Naturhist. Mus. Wien, 96A: 67-93.

BRZOBOHATY, R., 1995: Diaphus otoliths from the European Oligocene (Myctophidae, Teleostei). - Bulletin Van Het Koninklijk Belgisch Instituut Voor Natuurwetenschappen, Aarwetenschappen, 65: 257268.
BRZOBOHATY, R. \& NOLF, D., 1992: Fish otoliths as paleobathymetric indicators. Paleontologia i evolució, 24-25: 255-264 (1994).

BUSSING, W.A. \& LÓPEZ, M.I., 1993: Peces demersales y pelágicos costeros del Pacífico de Centro América Meridional. Guía ilustrada. - Rev. Biol. Tropical, Publ. Espec., 164 págs. (1994)

CAMPOS, L., 1987: Geología de Fila Asunción y zonas aledañas, Atlántico Central, Costa Rica. - 78 págs. Escuela Centroamericana de Geología , Universidad de Costa Rica (tésis inédita).

CARROLL, R.L. 1988: vertebrate paleontology and evolution. - W.H. Freeman Company, 698 págs.

CERVANTES, F., 1989: Base geológica para los análisis geotécnicos del Proyecto Hidroeléctrico Siquirres, Provincia de Limón, Costa Rica. - 117 págs. Escuela Centroamericana de Geología, Universidad de Costa Rica (tésis inédita).

CERVIGÓN, F. \& FISCHER, W., 1979: Catálogo de especies marinas de interés económico actual o potencial para América Latina. Parte I Atlántico centro y suroccidental. - Infopesca 372 págs.

CHIRICHIGNO, N., FISCHER, W. \& NAUEN, C.E., 1982: Catálogo de especies marinas de interés económico actual o potencial para América Latina. Parte II Pacífico centro y suroriental. - Infopesca 588 págs.

FERNÁNDEZ, J.A., 1987: Geología de la hoja topográfica Tucurrique (1:50000, I.G.N.C.R., N.34451). - 206 págs. Escuela Centroamericana de Geología, Universidad de Costa Rica, (tésis inédita).

FITCH, J.E., 1964: The fish fauna of the Playa del Rey Locality, a Southern California Marine Pleistocene Deposit. - Los Angeles County Museum, Contr.in Sci., 82: 135 . 
FITCH, J.E., 1966: Additional fish remains, mostly otoliths, from a Pleistocene deposit at Playa del Rey, California. - Los Angeles County Museum, Contr.in Sci., 119: 1-16.

FITCH, J.E., 1967: The marine fish fauna, based primarily on otoliths, of a Lower Pleistocene deposit at San Pedro, California (LACMIP 332, San Pedro Sand). - Los Angeles County Museum, Contr.in Sci., 128: 1-23.

FITCH, J.E., 1968: Otoliths and other fish remains from the Timms Point Silt (Early Pleistocene) at San Pedro, California. Los Angeles County Museum, Contr.in Sci., 146: 1-29.

FITCH, J.E., 1969: Fossil Lanternfish otoliths of California, with notes on fossil Myctophidae of North America. - Los Angeles County Museum, Contr.in Sci., 173: 1-20.

FITCH, J.E., 1970: Fish remains, mostly otoliths and teeth, from the Palos Verdes Sand (Late Pleistocene) of California. - Los Angeles County Museum, Contr.in Sci., 199: $1-41$.

FITCH, J.E. \& LAVENBERG, R.J., 1983: Teleost fish otoliths from Lee Creek Mine, Aurora, North Carolina (Yorktown Formation: Pliocene). - Smithsonian Contributions to Paleobiology, 53(1): 509-529.

GILLETTE, D.G., 1984: A marine ichthyiofauna from the Miocene of Panamá, and tertiary caribbean faunal province. - En: MacFadden, B. J. (ed.): Origin and evolution of the Cenozoic vertebrate fauna of Middle America. - Journal of Vertebrate Paleontology, 4(2): 172-186.

MORALES-NIN, B., 1991: Determinación del crecimiento de los peces óseos en base a la microestructura de los otolitos. - FAO Documento Técnico de Pesca, 322: 58 págs.

NOLF, D., 1980: Contribution a l'etude des otolithes des poissons III. Arguments nouveaux pour le rapprochement des poissons ophidioides et gadiformes. - Bull. Inst. r. Sci. Nat. Belg. Biologie, 52: 1-9.

NOLF, D., 1991: Geology and paleontology of the Eocene Drazinda Shale Member of the Khirthar Formation, central Western Pakistan, Part. III. Fish Otoliths. - Tertiary Research, 12(3-4): 121-126, 21 figs.

NOLF, D., 1995: Studies of fossil Otoliths -The State of the Art. - En: Secor, D.H.; Dean, J.M. \& Campana, S.E. (eds.): Recent developments in fish otolith research. - The Belle W. Baruch Library in Marine Science, 19: 513-544. Univ.of South Califoria Press.

NOLF, D. \& Brzobohaty, R., 1994: Fish otoliths from the Late Oligocene (Eger and Kiscell Formations) in the Eger area (northeastern Hungary). - Bulletin Van Het Koninklijk Belgisch Instituut Voor Natuurwetenschappen, Aarwetenschappen, 64: 225-252.

NOLF, D. \& DOCKERY III, D.T., 1993: Fish otoliths from the Matthews Landing Marl Member (Porters Creek Formation), Paleocene of Alabama. - Mississippi Geology, 14(2): 24-39.

NOLF, D. \& STEURBAUT, 1987: Description de la premiere fauna ichthyologique exclusivament bathyale du tertiaire d'Europe: otolithes de l'Oligocene Inférieur du gisement de Pizzocorno, Italie septentrionale. - Bulletin Van Het Koninklijk Belgisch Instituut Voor Natuurwetenschappen, Aarwetenschappen, 57: 217 230

NOLF, D. \& STRINGER, G.L., 1992: Neogene paleontology in the northern Dominican Republic. 14. Otoliths of teleostean fishes. - Bull. of Amer. Paleontology. 102(340): 41-81, láms. 9-17.

OLSSON, A., 1922: The Miocene of Northern Costa Rica. Bull. of Amer. Paleontology, 9(39): 179-46, láms.1-32. 
PIZARRO, D., 1987: Biostratigrafía de la Formación Uscari en base a foraminíferos planctónicos. - Rev. geol. América Central, 7: 1-63.

SPRECHMANN, P.; ASTORGA, A.; CALVO, C. \& FERNÁNDEZ, A., 1994: Stratigraphic chart of the sedimentary basins of
Costa Rica, Central América. - En: Seyfried \& Hellmann (eds.): Geology of an evolving island arc. - Profil, 7: 427-433.

STRINGER, G.L., 1992: Late Pleistocene-Early Holocene teleostean otoliths from a Mississippi River Mudlump. - J. Vertebrate Paleontology, 12 (1): 33-41. 